\title{
Association between susceptibility of diabetic nephropathy and gene polymorphisms in ELMO1 and IL-8: a systemic review and meta-analysis
}

Yanting Zhu

Shaanxi Provincial People's Hospital

Xiaoming Wang

Shaanxi Provincial People's Hospital

Yan Sun

Shaanxi Provincial People's Hospital

Qiong Wang

Shaanxi Provincial People's Hospital

\section{Bing Wu}

Shaanxi Provincial People's Hospital

Penghua Zhou

Shaanxi Provincial People's Hospital

Zhenjiang Li ( $\nabla$ m15191817651@163.com )

\section{Research article}

Keywords: Diabetic nephropathy, Gene polymorphism, ELM01, IL-8, Meta-analysis

Posted Date: October 1st, 2019

DOI: https://doi.org/10.21203/rs.2.15384/v1

License: (a) (1) This work is licensed under a Creative Commons Attribution 4.0 International License.

Read Full License 


\section{Abstract}

Background: The engulfment and cell motility 1 (ELM01) and interleukin-8 (IL8) gene polymorphisms have been previously implicated in diabetic nephropathy (DN) susceptibility. However, the results are inconsistent. We aimed to examine this issue by systematic meta-analysis.

Methods: An electronic search was conducted in PubMed, Web of Science, Embase, Cochrane Library, China National Knowledge Infrastructure and Wanfang Database to identify all the eligible studies. Odds ratios (ORs) with $95 \%$ confidence intervals (Cls) were calculated to investigate the associations. All statistical analyses were performed by STATA 12.0 .

Results: Of the 11 studies included, 9 studies were performed to detect EML01 rs741301 polymorphism; 5 studies were used to examine IL-8 rs4073 polymorphism. The results revealed no significant association between EMLO1 rs741301 polymorphism and DN risk. While, stratified analysis by ethnicity indicated that EML01 rs741301 polymorphism was associated with an increased risk of DN risk among Asians (GG vs. $G A+A A: O R=1.840,95 \% \mathrm{Cl}=1.338-2.529, \mathrm{P}=0.000 ; \mathrm{GG}$ vs. $\mathrm{AA}: \mathrm{OR}=1.834,95 \% \mathrm{Cl}=1.309-2.569, \mathrm{P}=0.000$; $\mathrm{G}$ vs. $\mathrm{A}: \mathrm{OR}=1.222,95 \% \mathrm{Cl}=1.053-1.417, \mathrm{P}=0.008$ ). As for IL-8 rs4073 polymorphism, a positive correlation between this gene polymorphism and DN risk was found (AA+AT vs. TT: OR=1.450, 95\% Cl= 1.166-1.802, $P=0.001 ; A T$ vs. $T T: O R=1.420,95 \% C l=1.129-1.786, P=0.003 ; A A$ vs. $T T: O R=1.553,95 \%$ $\mathrm{Cl}=1.094-2.203, \mathrm{P}=0.014 ; \mathrm{A}$ vs. $\mathrm{T}: \mathrm{OR}=1.291,95 \% \mathrm{Cl}=1.102-1.512, \mathrm{P}=0.002)$. After stratified population by ethnicity, the results in Caucasians remained significant (AA+AT vs. TT: OR=1.770, 95\% Cl=1.3542.315, $\mathrm{P}=0.000$; AT vs. $\mathrm{TT}: \mathrm{OR}=1.733,95 \% \mathrm{Cl}=1.304-2.302, \mathrm{P}=0.000 ; \mathrm{AA}$ vs. $\mathrm{TT}$ : $\mathrm{OR}=1.939,95 \% \mathrm{Cl}=$ 1.263-2.976, $\mathrm{P}=0.002 ; \mathrm{A}$ vs. $\mathrm{T}: \mathrm{OR}=1.494,95 \% \mathrm{Cl}=1.227-1.820, \mathrm{P}=0.000)$.

Conclusions: This meta-analysis indicates that the GG genotype of EMLO1 rs741301 polymorphism and the A allele of IL-8 rs4073 polymorphism might be risk factors for the development of DN.

\section{Background}

Diabetic nephropathy (DN), characterized by high disability rates and mortality rates, is a serious microvascular complication of diabetes mellitus (DM), leading to the occurrence of end-stage renal failure (ESRD)[1]. Although the exact pathogenesis of DN remains uncertain, it has become evident that environmental factors are likely to cooperate with genetic factors play a vital role in the development and progression of DN[2]. Recent genome-wide association studies (GWAS) have identified that several genetic single nucleotide polymorphisms (SNPs), which is involved in the pathogenesis of nephropathy, are associated with DN susceptibility, such as angiotensin I-converting enzyme (ACE) [3], angiotensin II type-1 receptor (AT1R)[4], vitamin D receptor (VDR)[5], engulfment and cell motility 1 (ELMO1)[6] and interleukin-8 (IL8)[7].

ELM01, a soluble cytoplasmic protein consisting of 720 amino acids, has been reported to be a keymediator in the pathogenesis of cytoskeletal rearrangements by interacting with the cytokinesis protein during cell motility and apoptotic cell phagocytosis[8]. It has been demonstrated that high glucose 
increases the expression of ELM01, which inhibits cell adhesion and promotes transcription growth factor- $\beta$ (TGF- $\beta$ ), collagen type 1 , fibronectin as well as integrin-linked kinase expressions to mediate the pathogenesis of $D N[9,10]$. ELMO1 gene, located on chromosome $7 p 14$, has been demonstrated to be a new candidate gene for DN in the first GWAS conducted in Japanese individuals[11]. Genetic studies have identified that rs741301 variants in the ELM01 gene appear to confer risk for DN[12].

The pro-inflammatory chemokine $\mathrm{IL}-8$, regulating inflammatory and immune processes, has been found to be highly expressed in urine of DN patients[13]. IL-8 gene, located on chromosome 4q13-q21, consists of 4 exons, 3 introns, and a promoter region[14]. It has been previously shown that rs4073 polymorphism of IL-8 gene participates in the development of DN among T2DM in the North and South Indian populations[7].

Recently, several genetic studies have reported the association between ELM01 rs741301 variant or IL-8 rs 4073 polymorphism and susceptibility of DN[7, 11, 12,15-18]. However, these results were controversial and inconsistencies because of the sample size, false positive results and different ethnicities in these publications. Therefore, we performed this meta-analysis to assess the pooled effect of ELM01 rs 741301 variant or IL-8 rs4073 polymorphism that have reproducibly been associated with DN.

\section{Methods}

\section{Literature search strategy}

The electronic literature search of PubMed, Web of Science, Embase, Cochrane Library, China National Knowledge Infrastructure (CNKI) and Wanfang Database were comprehensively performed for all the eligible studies published up to July 2019. The search terms were listed as follows: ("engulfment and cell motility 1" or "ELM01"), ("single nucleotide polymorphism" or "SNP" or "polymorphism" or "variation" or "mutation") and ("diabetic nephropathy") or ("interleukin-8" or "IL-8"), ("single nucleotide polymorphism" or "SNP" or "polymorphism" or "variation" or "mutation") and ("diabetic nephropathy"). Furthermore, the reference lists of all selected articles were manually conducted for further potentially eligible articles. The language, population, publication date, or type of report was not restricted and unpublished data were excluded.

\section{Inclusion and exclusion criteria}

The inclusion criteria were as follows: (1) case-control study; (2) investigating the association between EML01 rs741301 polymorphism or IL-8 rs4073 polymorphism and susceptibility of diabetic nephropathy; (3) diabetes mellitus (DM) was defined as control group; (4) complete data on the genotypic and allelic distributions of EML01 rs741301 polymorphism or IL-8 rs4073 polymorphism. The major exclusion criteria of this meta-analysis were: (1) not related to DN; (2) without control groups; (3) unavailable genotype information reported; (4) inappropriate article types (reviews, case reports, letters, or 
abstracts); (5) duplicated data. For overlapping studies, the largest or most recent publication was selected. All the potential studies were independently selected by two reviewers (Yanting Zhu and Qiong Wang).

\section{Data extraction}

The following information from each eligible study was independently extracted by two investigators (Yan Sun and Penghua Zhou) according to the standard protocol: first author, year of publication, country, ethnicity of each study population, duration of DM, number of cases and controls, genotyping method, genotype distribution in cases and controls and $P$ value for Hardy-Weinberg equilibrium (HWE) in controls.

\section{Statistical analysis}

STATA version 12.0 software (Stata Corporation, College Station, Texas, USA) was used to perform this meta-analysis. Chi-squared test was carried out to determine whether genotype frequencies in controls of each study were in accordance with Hardy-Weinberg equilibrium (HWE). P $<0.05$ was considered that control population in study was departed from HWE. Heterogeneity among the included studies was assessed using Chi-square-based $Q$ test and $P$ statistics. $P<0.10$ was considered that there was a statistical heterogeneity among the studies and the random-effect model was adopted for analysis. Otherwise, the fixed-effect model was applied for analysis as the pooling method $(P \geq 0.1)$. Sensitivity analysis of the overall population was tested by sequentially omitting one study in each turn. The combined odds ratio (OR) with its corresponding 95\% confidence interval (Cl) were calculated to evaluate the associations between EML01 rs741301 polymorphism or IL-8 rs4073 polymorphism and susceptibility of DN under five genetic models: recessive model (EML01 rs741301: GG vs. GA + AA; IL-8 rs4073: AA vs. AT + TT); dominant model (EML01 rs741301: GG + GA vs. AA; IL-8 rs4073: AA + AT vs. TT); codominant model (EML01 rs741301: GA vs. AA; IL-8 rs4073: AT vs. TT); homozygote model (EML01 rs741301: GG vs. AA; IL-8 rs4073: AA vs. TT); allele model (EML01 rs741301: G vs. A; IL-8 rs4073: A vs. T). Z-test was performed for assessing the significance of the pooled $O R$, with $P<0.05$ considered statistically significant. Publication bias was estimated by Begg's test and Egger's test and $P$ $<0.05$ was considered significant publication bias.

\section{Results}

\section{Literature search and study characteristics}

Initially, a total of 414 articles were identified to be relevant to the search terms from several different databases. After a systematical literature search according to the inclusion and exclusion criteria, 11 case-control studies from 7 published articles with a total of 2215 DN cases and 2001 DM controls investigating the genetic effects of EML01 rs741301 polymorphism and IL-8 rs4073 polymorphism on 
DN susceptibility, were recruited for this meta-analysis[7, 11, 12, 15-18]. A flow diagram in Figure 1 displayed the selection process with more particulars.

The main characteristics of included studies were summarized in Table 1. Of the 11 studies included, 9 studies from 6 selected articles containing 1879 cases and 1654 controls were performed to detect EML01 rs741301 polymorphism[11, 12, 15-18]; 5 studies (661 cases and 674 controls) in 2 published articles were used to examine IL-8 rs 4073 polymorphism[7, 17]. In terms of EML01 rs741301 polymorphism, 5 studies were performed in Asians[11, 15, 17], 4 studies was undertaken in Caucasians according to ethnicity $[12,16-18]$. As for IL-8 rs4073 polymorphism, there were 2 studies carried out of Asian ethnicity [17] and 3 studies conducted of Caucasian ethnicity[7, 17]. Genotype distributions and HWE examination results were exhibited in Table 2. The genotypes of EML01 rs741301 polymorphism in the control groups were in agreement with HWE excepted to 1 article[11]. For IL-8 rs4073 polymorphism, HWE deviation existed in control of 1 eligible study[7].

\section{Individual polymorphism meta-analysis and subgroup analysis}

The relationship between EML01 rs741301 polymorphism or IL-8 rs4073 variant and DN risk were shown in Table 3.

For EML01 rs741301 polymorphism, the susceptibility for DN did not show significant difference under the following genetic models: recessive model (GG vs. $G A+A A: O R=1.141,95 \% \mathrm{Cl}=0.941-1.385, \mathrm{P}=$ 0.179); dominant model (GG+GA vs. $A A: O R=0.999,95 \% \mathrm{Cl}=0.874-1.142, \mathrm{P}=0.990)$; codominant model (GA vs. AA: OR = 0.968, 95\% Cl = 0.840-1.115, $\mathrm{P}=0.650$ ); homozygote model (GG vs. AA: OR = $1.120,95 \% \mathrm{Cl}=0.911-1.378, \mathrm{P}=0.283)$ and allele model $(\mathrm{G}$ vs. $\mathrm{A}: \mathrm{OR}=1.033,95 \% \mathrm{Cl}=0.938-1.139, \mathrm{P}=$ 0.506). For studies assessing the relationship between EMLO1 gene polymorphism and DN risk in different ethnicity, significant increase in DN risk was identified in Asians under the recessive model (GG vs. $\mathrm{GA}+\mathrm{AA}: \mathrm{OR}=1.840,95 \% \mathrm{Cl}=1.338-2.529, \mathrm{P}=0.000$ ), the homozygote model (GG vs. $\mathrm{AA}: \mathrm{OR}=$ $1.834,95 \% \mathrm{Cl}=1.309-2.569, \mathrm{P}=0.000)$ and the allele model $(\mathrm{G}$ vs. $\mathrm{A}: \mathrm{OR}=1.222,95 \% \mathrm{Cl}=1.053-1.417$, $\mathrm{P}=0.008$ ) (Figure 2); no significant association was found among Asians in other genetic models (dominant model, $\mathrm{GG}+\mathrm{GA}$ vs. $\mathrm{AA}: \mathrm{OR}=1.121,95 \% \mathrm{Cl}=0.915-1.372, \mathrm{P}=0.271$ and codominant model, $\mathrm{GA}$ vs. $\mathrm{AA}: \mathrm{OR}=0.997,95 \% \mathrm{Cl}=0.805-1.234, \mathrm{P}=0.977)$. Available data did not suggest an association between this polymorphism and DN risk in Caucasians under all genetic models.

As for IL-8 rs4073 polymorphism, pooled effect among all studies suggested a significant association between the IL-8 rs4073 polymorphism and DN risk in dominant model (AA+AT vs. TT: OR $=1.450,95 \%$ $\mathrm{Cl}=1.166-1.802, \mathrm{P}=0.001)$; codominant model (AT vs. $\mathrm{TT}$ : $\mathrm{OR}=1.420,95 \% \mathrm{Cl}=1.129-1.786, \mathrm{P}=$ 0.003); homozygote model (AA vs. TT: $O R=1.553,95 \% \mathrm{Cl}=1.094-2.203, \mathrm{P}=0.014)$ and allele model $(\mathrm{A}$ vs. $\mathrm{T}: \mathrm{OR}=1.291,95 \% \mathrm{Cl}=1.102-1.512, \mathrm{P}=0.002)$; but not in recessive model (AA vs. AT +TT: $\mathrm{OR}=$ $1.272,95 \% \mathrm{Cl}=0.921-1.757, \mathrm{P}=0.144)$ (Figure 3 ). When stratified population by the basis of ethnicity, a 
positive correlation between this gene polymorphism and DN risk among Caucasians was found under the dominant model (AA+AT vs. $T T: O R=1.770,95 \% \mathrm{Cl}=1.354-2.315, \mathrm{P}=0.000)$, codominant model (AT vs. $T$ T: $O R=1.733,95 \% \mathrm{Cl}=1.304-2.302, \mathrm{P}=0.000$ ), homozygote model (AA vs. $\mathrm{TT}$ : $\mathrm{OR}=1.939$, $95 \% \mathrm{Cl}=1.263-2.976, \mathrm{P}=0.002)$ and allele model $(\mathrm{A}$ vs. $\mathrm{T}: \mathrm{OR}=1.494,95 \% \mathrm{Cl}=1.227-1.820, \mathrm{P}=$ 0.000); but not in recessive model ( $A A$ vs. $A T+T T: O R=1.419,95 \% \mathrm{Cl}=0.958-2.104, \mathrm{P}=0.081$ ) (Figure 4). However, IL-8 rs 4073 variant was not found to be associated with the risk of DN in Asians under all the genetic models.

\section{Sensitivity analysis}

In order to explore the stability of the crude results, sensitivity analysis was performed by sequentially omitting each study.

For EML01 rs741301 polymorphism, the result showed that the Bodhini's study influenced the stability of the pooled ORs. Therefore, a systematical meta-analysis was performed after deleting this study. The main result of the relationship between EML01 rs741301 polymorphism and diabetic nephropathy risk are presented in Table 4. Overall, the GG genotype of EMLO1 rs741301 polymorphism was a significantly increased risk factor for the development of DN (recessive model, GG vs. GA +AA: OR $=1.496,95 \% \mathrm{Cl}=$ $1.164-1.922, \mathrm{P}=0.002$; homozygote model, $\mathrm{GG}$ vs. $\mathrm{AA}: \mathrm{OR}=1.531,95 \% \mathrm{Cl}=1.172-1.999, \mathrm{P}=0.002$ and allele model, $\mathrm{G}$ vs. $\mathrm{A}: \mathrm{OR}=1.172,95 \% \mathrm{Cl}=1.039-1.321, \mathrm{P}=0.010)$. After categorizing subjects into different subgroups on the basis of ethnicity, the results in Asians remained significant. Exclusion of the sensitivity analysis-deviated studies did not meaningfully change the pooled estimates in Asians.

As for IL-8 rs 4073 polymorphism, the first study of Ahluwalia's altered the stability of the pooled ORs. After omitting this study, the results showed that a positive correlation was found between IL -8 rs 4073 polymorphism and DN in the overall population under the dominant model (AA+AT vs. TT: OR $=1.492$, $95 \% \mathrm{Cl}=1.132-1.966, \mathrm{P}=0.005$ ) and codominant model (AT vs. $\mathrm{TT}: \mathrm{OR}=1.622,95 \% \mathrm{Cl}=1.213-2.168$, $P=0.001)$. The subgroup analysis by ethnicity was not performed due to the limited data provided in Caucasians and Asians.

\section{Publication bias}

Publication bias was evaluated by Begg's funnel plot and Egger's linear regression test. All the shapes of the funnel plots were found to be symmetrical (Figure 5), indicating that there was a lack of publication bias for the association of EML01 rs741301 variant and IL-8 rs4073 polymorphism in all the genetic models (Table 5).

\section{Discussion}


Results of meta-analysis indicated that there was no significant association between EML01 rs741301 polymorphism and DN susceptibility in overall analyses. However, when stratified population by ethnicity, there was a significant increase on DN risk in Asians, but not in Caucasians. The GG genotype of EML01 rs741301 might be an increased risk factor for the development of DN. As for IL-8 rs4073 polymorphism, a significantly positive association between IL-8 rs4073 polymorphism and DN risk was found in the overall population. While after categorizing subjects into different subgroups on the basis of ethnicity, the results in Caucasians remained significant.

ELM01 functions as a guanine nucleotide exchange factor for the small GTPase Rac1 in combination with Dock180, leading to regulation of cell migration, cell motility and apoptotic cell phagocytosis[8, 19 , 20]. ELMO1 has been found to be over-expressed in serum of DN patients, which contributes to extracellular matrix (ECM) deposition, and thereby promotes the thickening of glomerular and tubular basement membrane, ultimately results in the occurrence and development of chronic renal injuries under high glucose concentration[10, 21]. Furthermore, recent study has demonstrated that inhibition of ELMO1 expression could be a promising option for slowing or preventing progression of the condition to ESRD[9]. Except for alteration of ELMO1 expression, its genetic polymorphism is also associated with the pathogenesis of DN[22-24]. The gene coding for ELM01, spans over 590kb on chromosome 7p14 and contains 22 exons and 21 introns[8]. A genome wide association study in Japan has identified that one SNP locus rs741301 in the 18th intron of the ELM01 gene was found to be strongly associated with DN[11]. In addition, later studies have demonstrated a significant association between rs741301 polymorphism and DN in South Indians[12], Chinese[15] and Iranian population[18]. In contrast, some other studies have shown no significant association between genetic variation in the ELMO1 rs741301 and DN[16]. To date, the effect of ELMO1 rs741301 polymorphism on DN risk has not been examined.

This present meta-analysis synthesized 9 studies from 6 selected articles containing 1879 cases and 1654 controls to detect the relationship between EML01 rs741301 polymorphism and DN. The pooled results suggested that no significant association between EML01 rs741301 polymorphism and DN susceptibility was found in overall analyses. When stratified population by ethnicity, analysis indicated that a significant increase on DN risk in Asians, but not in Caucasians. Furthermore, results showed that the Bodhini's study influenced the stability of the pooled ORs. Exclusion of the sensitivity analysisdeviated studies did not meaningfully change the pooled estimates in Asians.

$\mathrm{IL}-8$, a member of the C-X-C motif (CXC) subfamily of chemokines, is involved in angiogenesis, tumorigenesis, tissue invasion and metastasis by initiation and amplification of inflammation[25-27]. IL -8 has been found to be a major role in the pathogenesis of DN by promotion of oxidative stress and inflammatory response[28]. It is highly probable that IL-8 polymorphism, affecting the ability of individuals to produce IL-8, is associated with the development of DN[17]. IL-8 is encoded by the CXCL8 gene, which is located on chromosome $4 q-13-21$ and consists of a proximal promoter region, 4 exons, and 3 introns[14]. Recent study has showed a positive association between IL8 rs4073 polymorphism and DN in Indians, but not in Malays or Chinese[17]. Another study has suggested that IL-8 $-251 \mathrm{AA}$ 
genotype was more prevalent in North Indian DN group[7]. Therefore, this meta-analysis provided a statistical estimation to demonstrate the relationship between IL8 rs4073 variant and DN risk.

5 studies from 2 selected publications with a total of 661 cases and 674 controls were performed to investigate the correlation between IL8 rs 4073 variant and DN risk. A positive association was found between the IL -8 rs 4073 polymorphism and DN risk in overall population. After stratified population by ethnicity, the results in Caucasians remained significant. Moreover, Ahluwalia's first study altered the stability of the pooled ORs. After omitting this study, the results showed that a significantly increased risk of DN was found in overall population. The A allele of IL-8 rs4073 might be a risk factor for the development of DN.

There are several advantages in the present study. Firstly, the most recent literature was included. Secondly, the sensitivity analysis of each genetic model by sequentially deleting one study at a time was examined to improve the credibility of the results. However, this meta-analysis has some advantages.

First, original data in some studies was lacking, which might limit sufficient statistical power to clarify the effect of EML01 rs741301 polymorphism and IL-8 rs4073 variant on asthma risk. Second, higher heterogeneity was found in some pooled analyses, which might greatly affect the conclusions of metaanalysis. Some potentially relevant factors except for ethnicity such as age, sex, BMI and drug treatment status may influence the heterogeneity. Third, some studies employed DM only as the control group; whereas others studies used healthy people and DM as the controls, respectively. The difference between DN patients and healthy people in EML01 rs741301 polymorphism and IL-8 rs4073 variant was unable to evaluate due to limited availability of published data.

\section{Conclusion}

In conclusion, results of meta-analysis demonstrate that there was no significant association between EML01 rs741301 polymorphism and DN susceptibility in overall analyses. However, when stratified population by ethnicity, there was a significant increase on DN risk in Asians, but not in Caucasians. The GG genotype of EML01 rs741301 might be an increased risk factor for the development of DN. As for IL8 rs4073 polymorphism, a significantly positive association between IL-8 rs4073 polymorphism and DN risk was found in the overall population. While after categorizing subjects into different subgroups on the basis of ethnicity, the results in Caucasians remained significant.

\section{Abbreviations}

DN: diabetic nephropathy; DM: diabetes mellitus; ESRD: end-stage renal disease; GWAS: Genome-wide Association Study; SNPs: single nucleotide polymorphisms; ACE: angiotensin I-converting enzyme; AT1R: angiotensin II type-1 receptor; VDR: vitamin D receptor; ELM01: engulfment and cell motility 1 ; IL-8: interleukin-8; TGF- $\beta$ : transcription growth factor- $\beta$; CNKI: China National Knowledge Infrastructure; HWE: Hardy-Weinberg equilibrium; OR: odds ratio; Cl: confidence interval. 


\section{Declarations}

Funding

None.

\section{Acknowledgements}

None.

\section{Availability of data and material}

Not applicable.

\section{Authors' contributions}

Yanting Zhu: study protocol, literature search, data abstraction for meta-analysis, formulation of protocol, writing manuscript. Xiaoming Wang, Yan Sun, Qiong Wang, Bing Wu and Penghua Zhou: literature search, data abstraction for meta-analysis. Zhenjiang Li: study protocol, third reviewer of data abstraction, supervision of data abstraction, preparation of manuscript, submission for publication. All authors read and approved the final manuscript.

\section{Competing interests}

Authors declare no financial affiliations or conflicts of interest in submitting the study for publication.

\section{Consent for publication}

Not applicable.

\section{Ethics approval and consent to participate}

Not applicable.

\section{Author details}

Center of Nephropathy and Hemodialysis, Shaanxi Provincial People's Hospital, Xi'an, Shaanxi 710061, People's Republic of China. 


\section{References}

1. Collins AJ, Foley RN, Chavers B, Gilbertson D, Herzog C, Johansen K, et al. 'United States Renal Data System 2011 Annual Data Report: Atlas of chronic kidney disease \& end-stage renal disease in the United States. American journal of kidney diseases: the official journal of the National Kidney Foundation. 2012;59(1 Suppl 1):A7, e1-420.

2. Kanwar YS, Wada J, Sun L, Xie P, Wallner El, Chen S, et al. Diabetic nephropathy: mechanisms of renal disease progression. Experimental biology and medicine (Maywood, NJ). 2008;233(1):4-11.

3. Wyawahare M, Neelamegam R, Vilvanathan S, Soundravally R, Das AK, Adithan C. Association of Angiotensin-Converting Enzyme Gene Polymorphisms and Nephropathy in Diabetic Patients at a Tertiary Care Centre in South India. Clinical medicine insights Endocrinology and diabetes. 2017;10:1179551417726779.

4. Ali Z, Kusrini I, Shahab A, Saleh I. Association between A1166C Polymorphism of the Angiotensin II Type-1 Receptor Gene and Type-2 Diabetic Nephropathy in an Indonesian Malay Population. Acta medica Indonesiana. 2018;50(4):314-9.

5. Razi F, Meshkani MA, Zarrabi F, Sadr M, Asgarbeik S, Bandarian F, et al. Haplotypes in vitamin D receptor gene encode risk in diabetic nephropathy. Gene. 2019;683:149-52.

6. Pezzolesi MG, Katavetin P, Kure M, Poznik GD, Skupien J, Mychaleckyj JC, et al. Confirmation of genetic associations at ELMO1 in the GoKinD collection supports its role as a susceptibility gene in diabetic nephropathy. Diabetes. 2009;58(11):2698-702.

7. Ahluwalia TS, Khullar M, Ahuja M, Kohli HS, Bhansali A, Mohan V, et al. Common variants of inflammatory cytokine genes are associated with risk of nephropathy in type 2 diabetes among Asian Indians. PloS one. 2009;4(4):e5168.

8. Gumienny TL, Brugnera E, Tosello-Trampont AC, Kinchen JM, Haney LB, Nishiwaki K, et al. CED12/ELMO, a novel member of the Crkll/Dock180/Rac pathway, is required for phagocytosis and cell migration. Cell. 2001;107(1):27-41.

9. Hathaway CK, Chang AS, Grant R, Kim HS, Madden VJ, Bagnell CR, Jr., et al. High Elmo1 expression aggravates and low Elmo1 expression prevents diabetic nephropathy. Proceedings of the National Academy of Sciences of the United States of America. 2016;113(8):2218-22.

10. Shimazaki A, Tanaka Y, Shinosaki T, Ikeda M, Watada H, Hirose T, et al. ELMO1 increases expression of extracellular matrix proteins and inhibits cell adhesion to ECMs. Kidney international. 2006;70(10):1769-76.

11. Shimazaki A, Kawamura Y, Kanazawa A, Sekine A, Saito S, Tsunoda T, et al. Genetic variations in the gene encoding ELMO1 are associated with susceptibility to diabetic nephropathy. Diabetes. 2005;54(4):1171-8.

12. Bodhini D, Chidambaram M, Liju S, Revathi B, Laasya D, Sathish N, et al. Association of rs 11643718 SLC12A3 and rs741301 ELM01 Variants with Diabetic Nephropathy in South Indian Population. Annals of human genetics. 2016;80(6):336-41. 
13. Tashiro K, Koyanagi I, Saitoh A, Shimizu A, Shike T, Ishiguro C, et al. Urinary levels of monocyte chemoattractant protein-1 (MCP-1) and interleukin-8 (IL-8), and renal injuries in patients with type 2 diabetic nephropathy. Journal of clinical laboratory analysis. 2002;16(1):1-4.

14. Mukaida N, Shiroo M, Matsushima K. Genomic structure of the human monocyte-derived neutrophil chemotactic factor IL-8. Journal of immunology (Baltimore, Md: 1950). 1989;143(4):1366-71.

15. Wu HY, Wang Y, Chen M, Zhang X, Wang D, Pan Y, et al. Association of ELMO1 gene polymorphisms with diabetic nephropathy in Chinese population. Journal of endocrinological investigation. 2013;36(5):298-302.

16. Yadav AK, Kumar V, Dutta P, Bhansali A, Jha V. Variations in CCR5, but not HFE, ELM01, or SLC12A3, are associated with susceptibility to kidney disease in north Indian individuals with type 2 diabetes. Journal of diabetes. 2014;6(6):547-55.

17. Yahya MJ, Ismail PB, Nordin NB. Association of CCL2, CCR5, ELMO1, and IL8 Polymorphism with Diabetic Nephropathy in Malaysian Type 2 Diabetic Patients. 2019;2019:2053015.

18. Mehrabzadeh M, Pasalar P, Karimi M, Abdollahi M, Daneshpour M, Asadolahpour E, et al. Association between ELMO1 gene polymorphisms and diabetic nephropathy in an Iranian population. Journal of diabetes and metabolic disorders. 2015;15:43.

19. Brugnera E, Haney L, Grimsley C, Lu M, Walk SF, Tosello-Trampont AC, et al. Unconventional Rac-GEF activity is mediated through the Dock180-ELMO complex. Nature cell biology. 2002;4(8):574-82.

20. Elliott MR, Zheng S, Park D, Woodson RI, Reardon MA, Juncadella IJ, et al. Unexpected requirement for ELMO1 in clearance of apoptotic germ cells in vivo. Nature. 2010;467(7313):333-7.

21. Yang $C$, Sorokin A. Upregulation of fibronectin expression by COX-2 is mediated by interaction with ELM01. Cellular signalling. 2011;23(1):99-104.

22. Kim S, Abboud HE, Pahl MV, Tayek J, Snyder S, Tamkin J, et al. Examination of association with candidate genes for diabetic nephropathy in a Mexican American population. Clinical journal of the American Society of Nephrology: CJASN. 2010;5(6):1072-8.

23. Leak TS, Perlegas PS, Smith SG, Keene KL, Hicks PJ, Langefeld CD, et al. Variants in intron 13 of the ELM01 gene are associated with diabetic nephropathy in African Americans. Annals of human genetics. 2009;73(2):152-9.

24. Hanson RL, Millis MP, Young NJ, Kobes S, Nelson RG, Knowler WC, et al. ELMO1 variants and susceptibility to diabetic nephropathy in American Indians. Molecular genetics and metabolism. 2010;101(4):383-90.

25. Ju D, Sun D, Xiu L, Meng X, Zhang C, Wei P. Interleukin-8 is associated with adhesion, migration and invasion in human gastric cancer SCG-7901 cells. Medical oncology (Northwood, London, England). 2012;29(1):91-9.

26. Ding S, Tang Z. IL-8 Is Involved in Estrogen-Related Receptor alpha-Regulated Proliferation and Migration of Colorectal Cancer Cells. 2017;62(12):3438-46.

27. Konno H, Ohta M, Baba M, Suzuki S, Nakamura S. The role of circulating IL-8 and VEGF protein in the progression of gastric cancer. Cancer science. 2003;94(8):735-40. 
28. Aydin M, Ozkok E, Ozturk O, Agachan B, Yilmaz H, Yaylim I, et al. Relationship between interleukin-8 and the oxidant-antioxidant system in end-stage renal failure patients. Experimental and clinical transplantation: official journal of the Middle East Society for Organ Transplantation. 2007;5(1):6103.

\section{Tables}

Due to technical limitations, all Table(s) are only available as a download in the supplemental files section.

\section{Table S1 Caption}

Meta-analysis of EML01 rs741301 and IL-8 rs4073 polymorphisms and DN susceptibility in which studies influencing the stability of the pooled ORs were deleted.

$\mathrm{OR}$, odds ratio; $\mathrm{Cl}$, confidence interval; $\mathrm{R}$, random effect model; $\mathrm{F}$, fixed effect model.

\section{Figures}




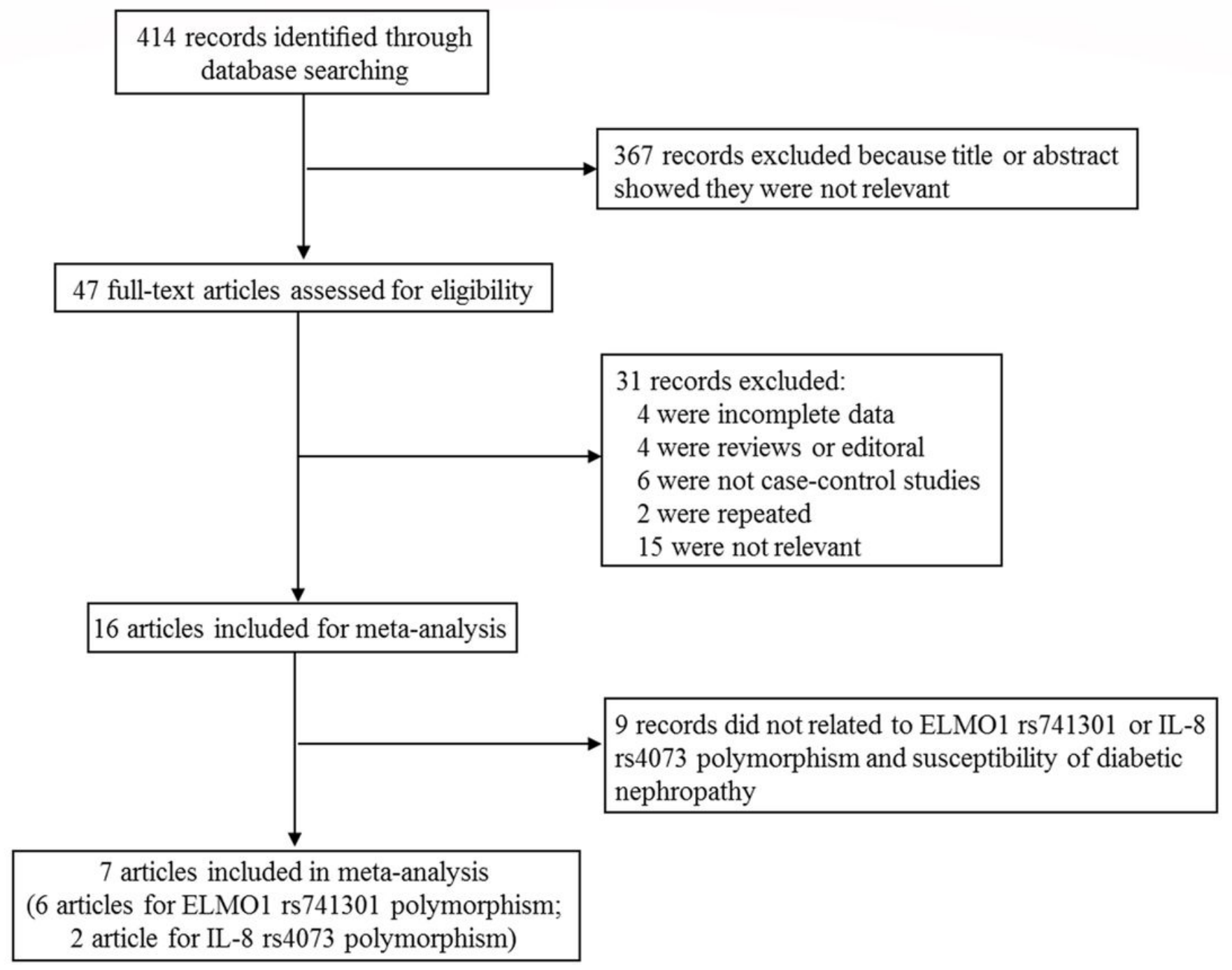

\section{Figure 1}

Flowchart of study selection procedure. 

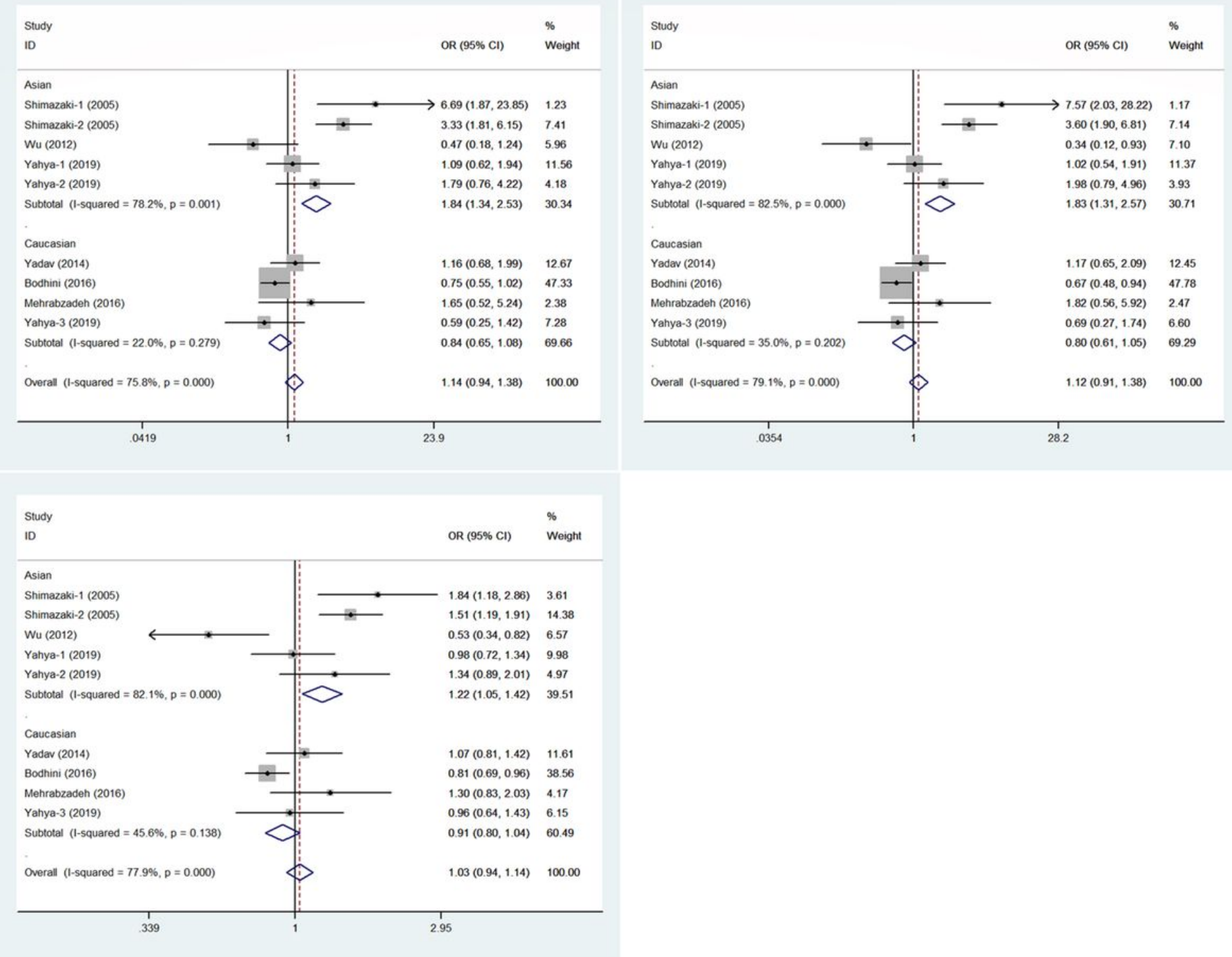

\section{Figure 2}

Forest plot of the association between EML01 rs741301 polymorphism and DN risk by ethnicity stratification under ( $A$ ) the recessive model (GG vs. GA+AA), (B) the homozygote model (GG vs. $A A$ ) and (C) the allele model ( $G$ vs. $A)$. 


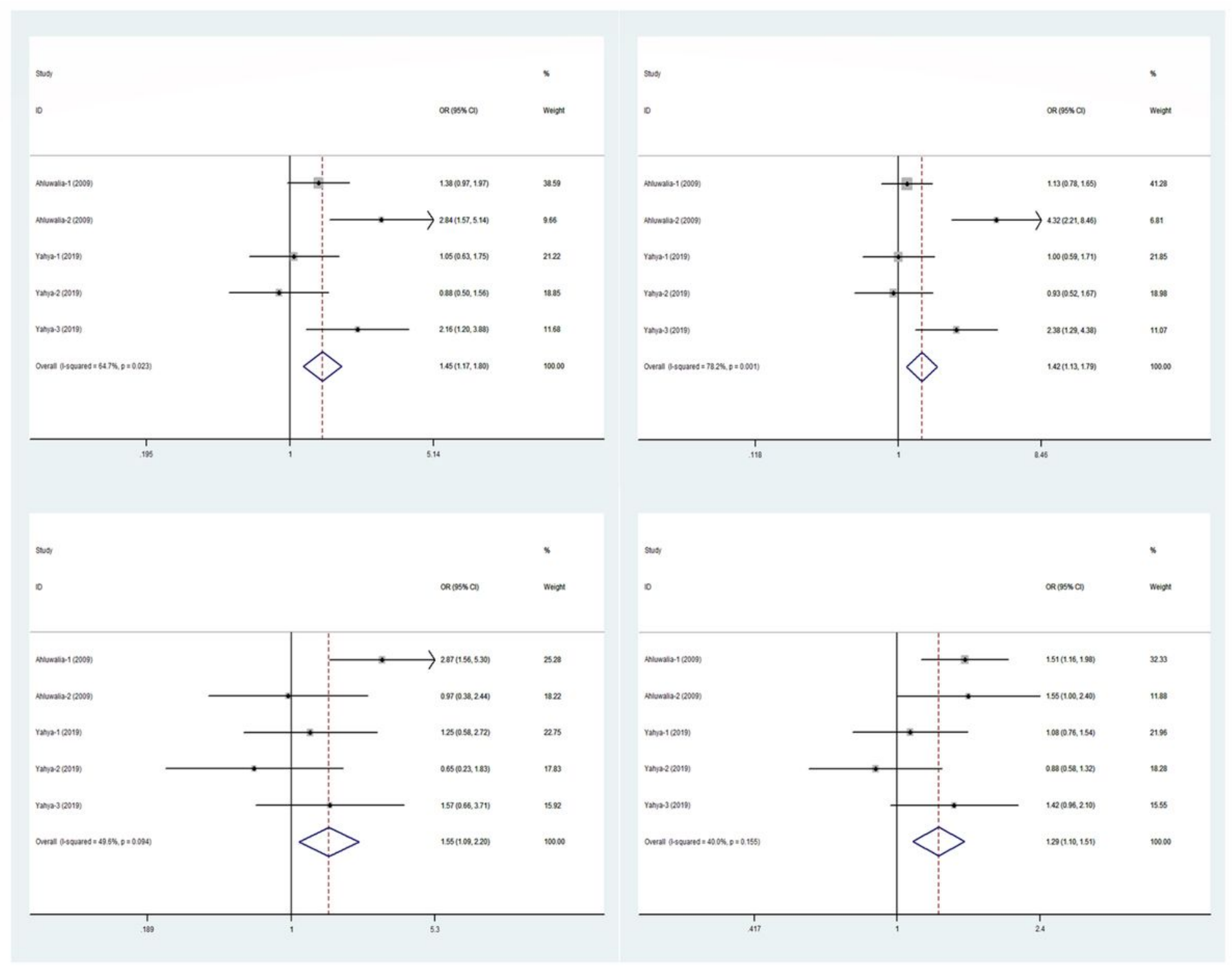

\section{Figure 3}

Forest plot of the association between IL-8 rs4073 polymorphism and DN risk under ( $A$ ) the dominant model (AA+AT vs. TT), (B) the codominant model (AT vs. TT), (C) the homozygote model (AA vs. TT) and (D) the allele model (A vs. T). 


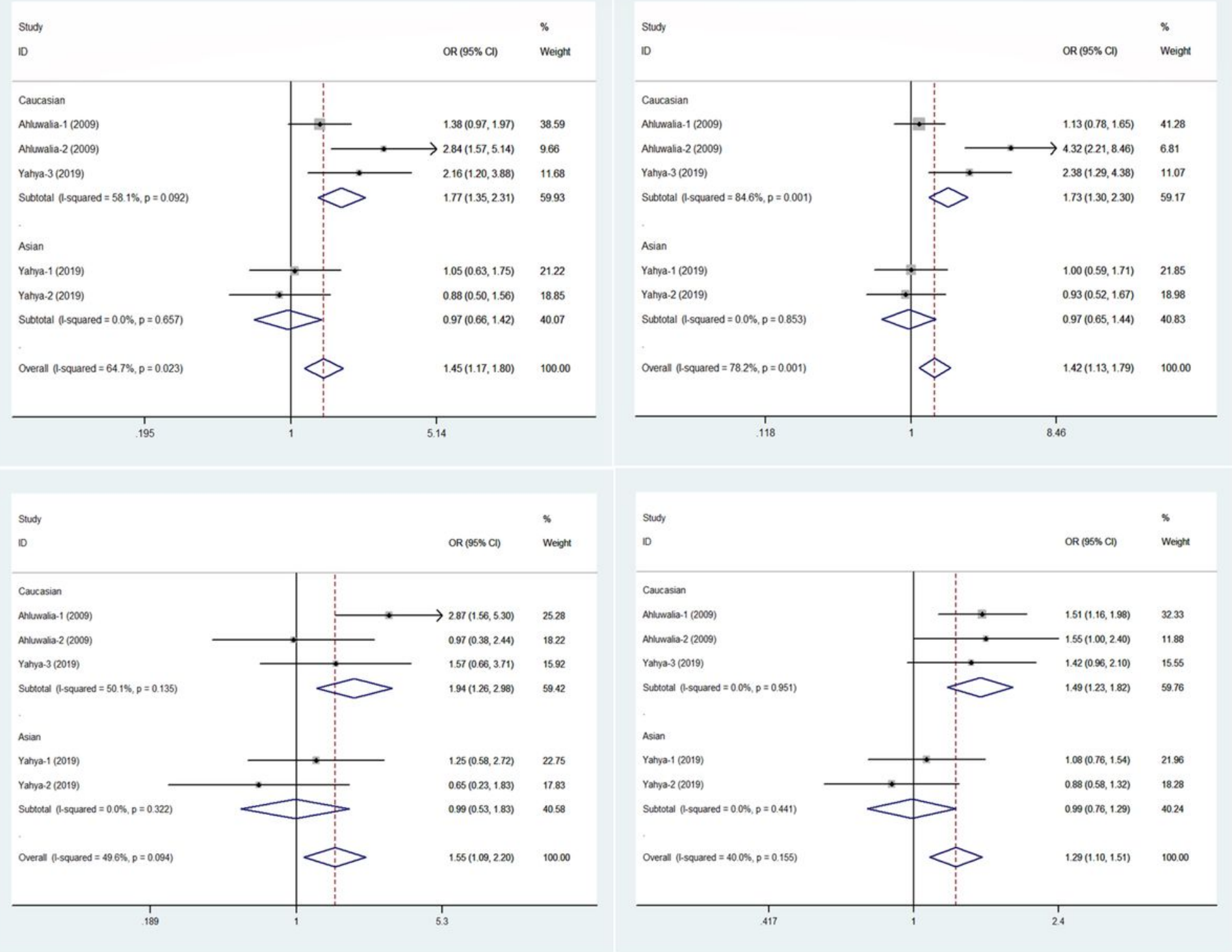

\section{Figure 4}

Forest plot of the association between IL-8 rs4073 polymorphism and DN risk stratified by ethnicity under(A) the dminant model (AA+AT vs. TT), (B) the codominant model (AT vs. TT), (C) the homozygote model (AA vs. TT) and (D) the allele model (A vs. T). 


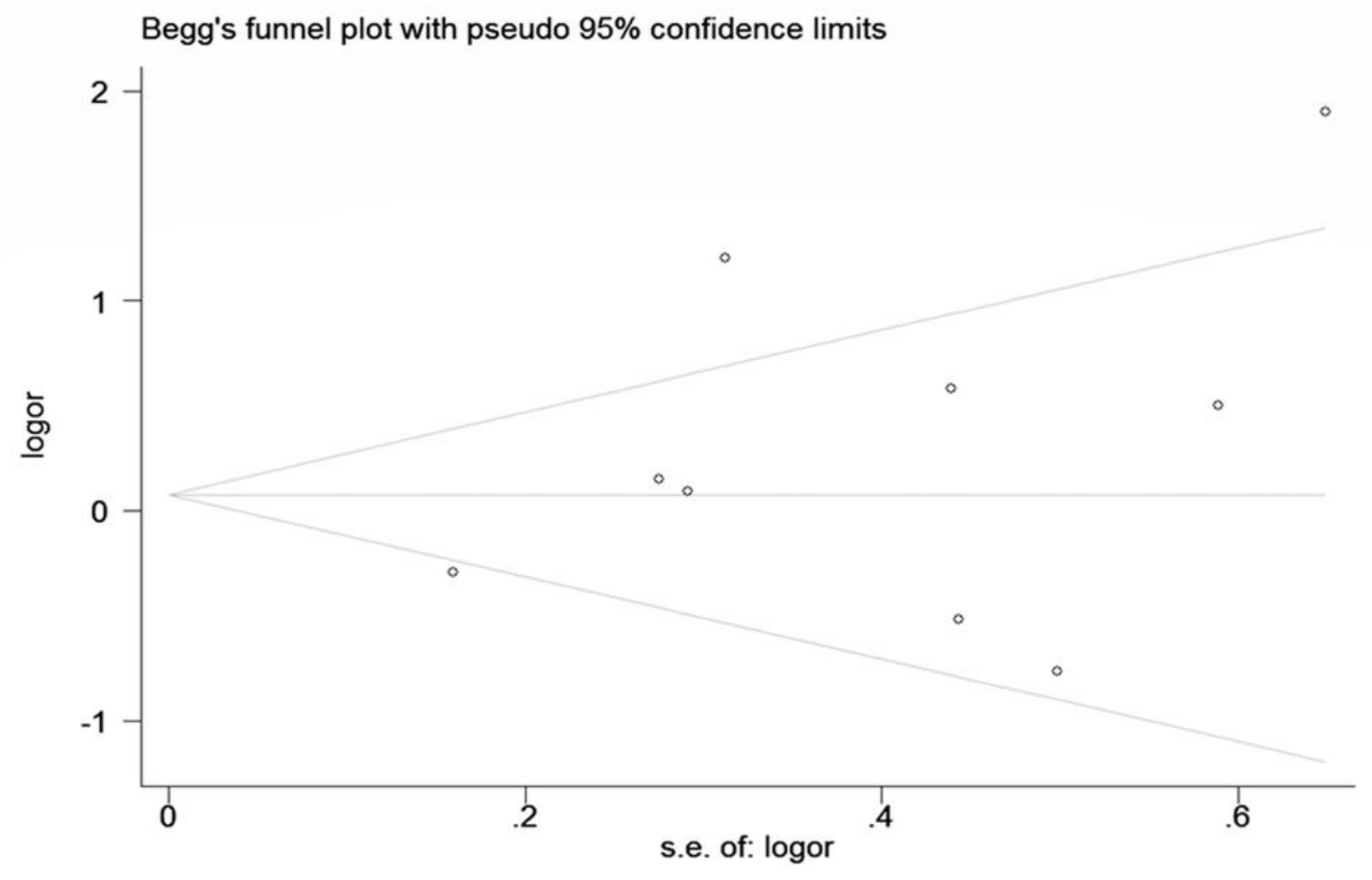

Begg's funnel plot with pseudo $95 \%$ confidence limits

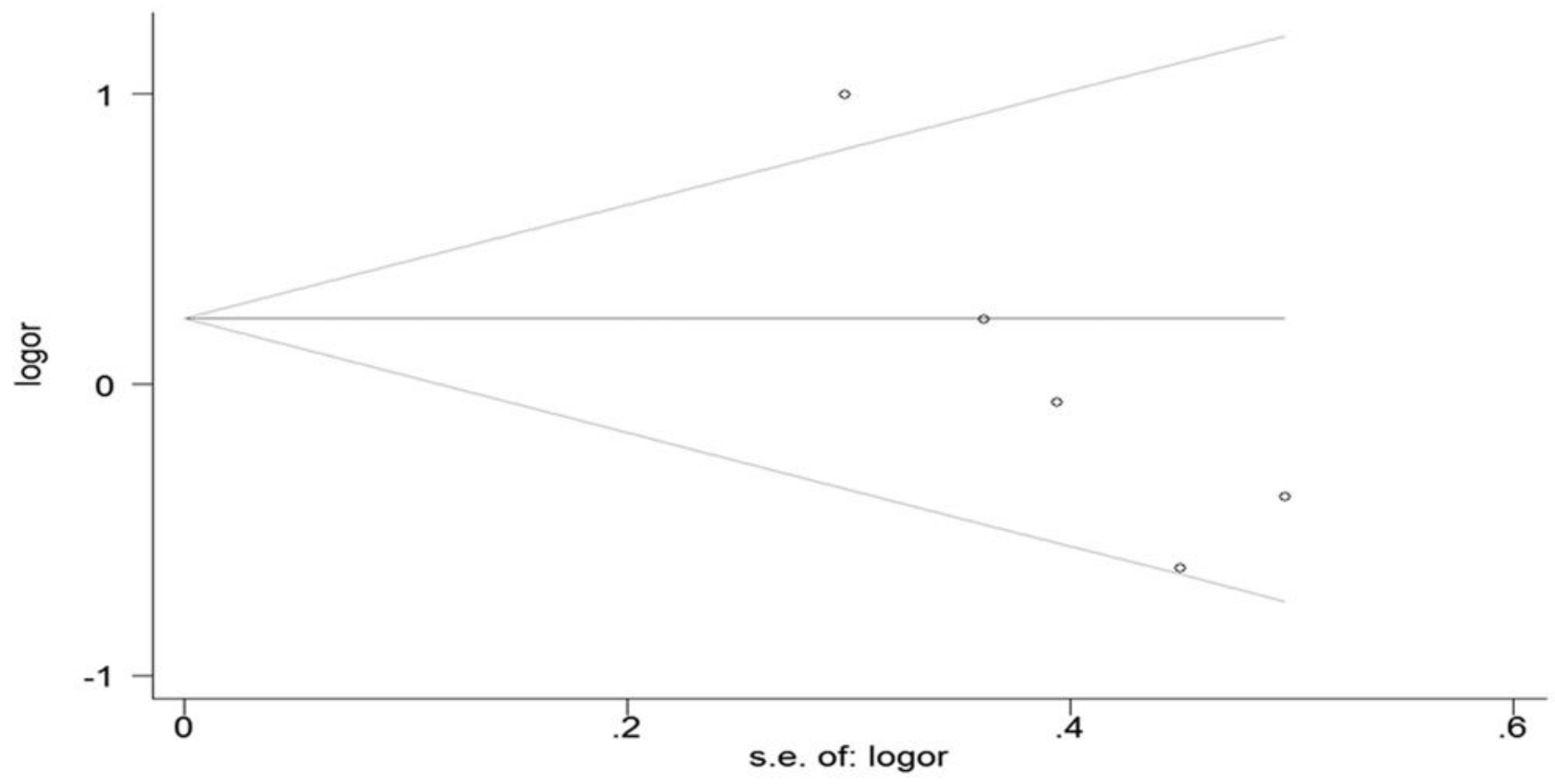

\section{Figure 5}

Begg's funnel plot of publication bias for the association between EML01 rs741301 and IL-8 rs4073 polymorphisms and DN risk under the recessive models (GG vs. GA+AA; AA vs. AT+TT).

\section{Supplementary Files}


This is a list of supplementary files associated with this preprint. Click to download.

- table3.tif

- table1.tif

- supplementaltable.tif

- table4.tif

- table2.tif 